\title{
Cytokines as markers of oncogenesis and therapy efficiency in chemically induced breast cancer
}

\author{
Poveshchenko Alexandr \\ Research Institute of Clinical \\ andExperimental Lymphology - Branch \\ of the Institute of Cytology and \\ Genetics, Siberian Branch of the \\ Russian Academy of Sciences \\ Novosibirsk, Russia \\ poveshchenkoa200@mail.ru \\ Kabakov Alexey \\ Research Institute of Clinical and \\ Experimental Lymphology - Branch of \\ the Institute of Cytology and Genetics, \\ Siberian Branch of the Russian \\ Academy of Sciences \\ Novosibirsk, Russia \\ doctor03-85@ngs.ru
}

\author{
Kazakov Oleg \\ Research Institute of Clinical and \\ Experimental Lymphology - Branch of \\ the Institute of Cytology and \\ Genetics, Siberian Branch of the \\ Russian Academy of Sciences \\ Novosibirsk, Russia \\ kazakoff_oleg@mail.ru \\ Strunkin Dmitry \\ Research Institute of Clinical and \\ Experimental Lymphology - Branch of \\ the Institute of Cytology and Genetics, \\ Siberian Branch of the Russian \\ Academy of Sciences \\ Novosibirsk, Russia \\ Strunkind@mail.ru
}

\author{
Orlov Nikolai \\ Research Institute of Clinical and \\ Experimental Lymphology - Branch of \\ the Institute of Cytology and Genetics \\ Siberian Branch of the Russian \\ Academy of Sciences \\ Novosibirsk, Russia \\ nbo700@mail.ru \\ Vladimir Konenkov \\ Research Institute of Clinical and \\ Experimental Lymphology - Branch of \\ the Institute of Cytology and \\ Genetics, Siberian Branch of the \\ Russian Academy of Sciences \\ Novosibirsk, Russia \\ vikonenkov@gmail.com
}

\begin{abstract}
Breast cancer was induced by the administration of n-methyl-N-nitrosourea to Wistar rats. Some animals underwent only surgical intervention or only polychemotherapy (cyclophosphamide, methotrexate, 5-ftorouracil). In some animals, both types of therapy were combined, and in a separate group, the administration of the Panagen drug, which was a fragmented DNA molecule, was added to polychemotherapy. To study the concentration of cytokines in lymph, we used the BioPlex Pro Rat Cytokoness 24-Plex Assay test system (Bio-Rad, USA). It was shown that in rats with breast cancer the content in the lymph and blood of most of the studied cytokines such as

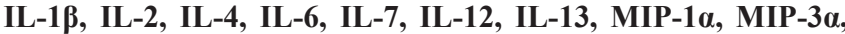
RANTES, TNF- $\alpha$, MCP-1 were statistically significantly higher, and IL-10 and GRO / KC, lower than in intact animals. Surgical removal of the tumor led to a significant decrease in the content of both pro-inflammatory and anti-inflammatory cytokines in the lymph. PCT led to a significant decrease in the content of IL$1 \beta$, IL-4, IL-6, IL-7, MIP-1 $\alpha$, MIP-3 $\alpha$, RANTES in the blood serum and lymph of rats with breast cancer.
\end{abstract}

Keywords - breast cancer, Wistar rat, cytokines

Introduction

Breast cancer $(\mathrm{BC})$ maintains a leading position among oncological pathologies in women around the world $[1,2]$. The purpose of the study is to determine the quantitative characteristics and qualitative features of the blood and lymph cytokine profile at various stages of breast cancer oncogenesis, to conduct a comparative study of lymph cytokines and blood serum of Wistar rats after surgery, polychemotherapy, polychemotherapy in combination with the Panagen therapy.

\section{Materials and Methods}

The studies were carried out on sexually mature ( 3 months old) female rats of the Wistar breed weighing 250-300 $\mathrm{g}$ in the amount of 9-10 animals in each group: group 1 - intact rats; 2nd group - rats with breast cancer (tumor carriers); 3rd group - rats with breast cancer after polychemotherapy (PCT); Group 4 - rats after removal of breast cancer (operated animals), Group 5 - rats after removal of breast cancer and PCT, group 6 - rats after removal of breast cancer in combination with PCT and administration of the panagen preparation (fragmented DNA). BC was induced by the introduction of N-methyl-N-nitrosourea (MNU) 5 times with an interval of 7 days, subcutaneously in the region of the 2 nd breast on the right.,resulting in the formation of breast cancer (adenocarcinoma) after 6 months [1]. Surgical treatment was performed 6 months after the induction of breast cancer. For PCT, the CMF regimen was used (cyclophosphamide - at a dose of $100 \mathrm{mg} / \mathrm{m} 2$ from 1 to 14 days; methotrexate - at a dose of $40 \mathrm{mg} / \mathrm{m} 2$ on days 1 and 8; 5-ftoruracil - at a dose of $600 \mathrm{mg} / \mathrm{m} 2$ in 1 and 8) (Sigma-Aldrich, USA)]. The course of Panagen (fragmented DNA $(5 \mathrm{mg} / \mathrm{kg}$ ) therapy was carried out by intraperitoneal administration once for 14 days 3 hours after cyclophosphamide administration. The experiments used the substance of the Panagen isolated from human placenta. Lymph was taken from the thoracic lymphatic duct [1]. Concentrations of 24 cytokines in lymph were evaluated by flow fluorimetry on a 2-beam laser automated analyzer (BioPlex Assay System (Bio-Rad, USA) using a commercial test system (determined dynamic range of 2-32000 pkg / $\mathrm{ml}$ ) in accordance with manufacturer's instructions Bio-Plex Pro Rat Cytokoness 24-Plex Assay (Bio-Rad, USA).Differences between the groups were considered significant at $\mathrm{p}<0.05$. Significance of the differences was calculated by U-criterion the Mann-Whitney.

\section{Results}

In rats with breast cancer, the content of most of the studied cytokines such as IL-1 $\beta$, IL-2, IL-4, IL-6, IL- 7, IL-12, IL-13, IL-17A, IFN- $\gamma$, MIP-1 $\alpha$, MIP-3 $\alpha$, RANTES, TNF- $\alpha$, MCP-1 was statistically significantly higher in serum and lymph than in intact animals. According to many researchers, pro-inflammatory cytokines inside the tumor and its microenvironment are associated with the progression of breast cancer $[1,2]$. The high content of these biologically active factors in animals with breast cancer allows them to be attributed to tumor growth markers. Induction of breast cancer 
led to a decrease in the production of anti-inflammatory cytokine IL-10, which can contribute to immunosuppression and weakening of antitumor defense.

Surgical removal of the tumor resulted in a significant decrease in serum and lymph levels, such as pro-inflammatory cytokines (IL-1 $\beta$, IL-2, IL-12, IL-18, TNF- $\alpha$ ), chemokines (MIP-1 $\alpha$, MIP-3 $\alpha$, RANTES , GRO / KC) and antiinflammatory cytokines IL-4, IL-13, compared with breast cancer.

A comparative study of the cytokine content after removal of the tumor with intact animals showed that the content of IL10, IL-18, EPO, GRO / KC, RANTES was significantly higher in the group of control animals. Moreover, the level of production of IL-10 and EPO after surgery was comparable with the level in breast cancer. The main source of IL-10 in the tumor microenvironment is macrophages, which increase the expression of IL-10 in response to signals from dying cancer cells. Removal of the primary tumor site obviously weakened the signaling pathways, and IL-10 production was low. The content of IL-6, TNF- $\alpha$, and MCP-1 chemokine in the group of operated animals was significantly higher in comparison with the control, which allows us to consider them not only pro-inflammatory markers, but also metastasis markers.

PCT led to a significant decrease in the content of IL-1 $\beta$, IL-4, IL-6, IL-7, IL-12, IL-18, MIP-1 $\alpha$, MIP-3 $\alpha$, RANTES in the blood serum and lymph of rats with breast cancer. When studying the concentration of cytokines in the blood serum of rats after tumor removal, compared with animals after removal of breast cancer and PCT, no significant differences were found. Nevertheless, the content of IL-2, IL-5, IL-7, IL13, IL-17A, G-CSF, MCP-1 in the blood serum was significantly higher in the group of operated animals compared with the combined therapy. In this case, there are no changes in the production of metastatic markers such as IL$1 \beta$, IL-6, MIP-1 $\alpha$, MIP-3 $\alpha$, VEGF, MCP-1. But at the same time, the level of production of the chemokines GRO / KC and RANTES is higher with a combination of PCT and surgical treatment. Obviously, GRO / KC and RANTES determine not only metastasis, but also chemotaxis of immunocompetent cells.

The concentration of cytokines in the blood serum of the operated animals after PCT and administration of the Panagen significantly increased compared to the group of rats after surgical removal of breast cancer and PCT for most of the studied cytokines: IL-2, IL-4, IL-5, IL6, IL-7, IL-10, IL-12, IL-17A, IL-18, G-CSF, GM-CSF, GRO / KC, IFN $\gamma$, MIP-1 $\alpha$, MIP-3 $\alpha$. A study of the cytokine content in the lymph of the operated animals after polychemotherapy and the administration of the panagen drug also showed that most indicators of the cytokine content such as IL-5, IL-6, IL-7, IL10, IL-13, IL-17A, IL- 18, GRO / KC, IFN $\gamma$, MIP-3 $\alpha$ in lymph was also higher after administration of panagen. The stimulating effect of the drug Panagen on lymphoid cells is expressed in an increase in the production of a number of cytokines. This coincides with the notion that cytostatics in combination with Panagen possibly provide an antitumor effect and stimulate lymphoid cells of the immune system [3].

\section{Conclusion}

Our comparative study of the cytokine profile using a test system consisting of 24 cytokines of blood serum and lymph of Wistar rats found that the quantitative content of cytokines in breast cancer depends on the type of treatment for the disease. The production of cytokines may change in the process of progression, inhibition of tumor development or metastasis.

Thus, lymph and serum cytokines in experimental breast cancer are markers of tumor growth and metastasis, their levels depend on the type of treatment.

\section{REFERENCES}

[1] A.F. Poveshchenko, O.V. Kazakov, N.B. Orlov, O.V. Poveshchenko I.I. Kim, N.A. Bondarenko, I.G. Solovieva, D.N.Strunkin, A.V. Kabakov, T.V. Reiter, A.P. Lykov, S.S. Bogachev, E.A. Pokushalov V.I. Konenkov. Lymphatic cytokines as markers of oncogenesis and treatment efficacy in experimental Wistar rat mammary tumor. Pathological physiology and experimental therapy. 2016. 60(3): 68 75

[2] Sosnina A.V., Velikaya N.V., Autenshlus A.I. The role of cytokines in the pathogenesis of malignant neoplasms. Novosibirsk: Vector-Best, 2013, 80. (In Russian).

[3] E.A. Alyamkina, O.Y.Leplina, LV.Sakhno, E.R. Chernykh, A.A.Ostanin, Y.R. Efremov, A.G.Shilov, A.S. Proskurina, K.E. Orishchenko, E.V. Dolgova, V.A. Rogachev, V.P. Nikolin, N.A. Popova, S.N. Zagrebelniy, S.S. Bogachev, M.A. Shurdov. Effect of double-stranded DNA on maturation of dendritic cells in vitro. Cellular Immunology. 2010. 266: 46-51 